\title{
Das grosse Würfeln
}

\author{
Erhard Taverna
}

Britische Familienärzte sind die wichtigsten Grundversorger und stellen rund die Hälfte aller Mediziner des National Health Service (NHS). Fast alle Einwohner des Landes sind bei einem für sie verantwortlichen Familienarzt registriert. Dieser bezieht den grössten Teil seines vertraglich gesicherten Einkommens über diese NHSPatienten. Dieser Vertrag, bekannt als «General Medical Services Contract», besteht seit 1948 und wurde erst zweimal, 1966 und 1990, in grösserem Stil erneuert.

\section{«Qualität» durch Belohnung}

Seit einem halben Jahr existiert ein neues Abkommen mit der Regierung, die das bestehende Budget für Grundversorger um 20\%, entsprechend rund 2,2 Milliarden Franken, erhöht. War noch in den 80er Jahren ein ähnliches Vorhaben am Widerstand der Ärzte gescheitert, die ihre Qualität nicht für messbar hielten, führten die Resultate der evidenzbasierten Medizin in den 90ern zu einem Meinungsumschwung. Beide Seiten einigten sich auf gemeinsame Definitionen von Qualität und entsprechende Messmethoden. Der «Health Policy Report» im «New England Journal of Medicine» spricht von einem enormen «change in the culture of the profession», einem Wechsel, der hauptsächlich durch Forschungsarbeiten in den USA und im UK ermöglicht worden sei. Es war auch den Ärzten klar, dass ein System, das Messergebnisse finanziell belohnt, Gewinner und Verlierer produziert. Erst die massive Erhöhung der dafür vorgesehenen Geldmittel, die zunehmende öffentliche Kritik an den staatlichen Gesundheitsdiensten und Vergleiche mit anderen europäischen Ländern führten zu einem Durchbruch in den Vertragsverhandlungen. Damit verbunden waren auch grundlegende Konzessionen des NHS, zum Beispiel keine Dienstpflicht mehr ausserhalb der normalen Bürozeiten und zusätzliche Vergütungen für Sonderleistungen, wie die Betreuung von Süchtigen. Die Verhandlungen waren stets von Spezialisten des allgemein anerkannten «National Institute of Clinical Excellence» (www. nice.nhs.uk) begleitet, die Leitlinien und Prozesskomponenten erarbeitet haben.

Familienpraktiker können nun 1050 Punkte jährlich zusätzlich gewinnen, wenn sie allen
Variablen maximal genügen. Die Qualität der klinischen Arbeit berücksichtigt 10 chronische Krankheitszustände mit maximal 550 Punkten: Koronare Herzkrankheiten (121 Punkte), zerebrale Insulte (31), Hypertonie (105), Hyperthyreoidismus (8), Diabetes (99), mentale Störungen (41), COPD (45), Asthma (72), Epilepsie (16) und Krebs (12). Jedem dieser Zustände sind Indikatoren zugeordnet, deren Punktezahl wiederum von der Praxisgrösse und der Patientendurchmischung abhängig ist. Punkte lassen sich auch mit regelmässigen Kontrollen von Blutdruck und Cholesterin gewinnen, vor allem, wenn das vorgegebene Behandlungsziel erreicht wird. Patienten, die Kontrollen und Behandlungen ablehnen, können ausgeschlossen werden, wobei ein jährlicher Besucher des «Primary Care Trust», der für ein Gebiet von bis zu 100 Praxisbetrieben zuständig ist, die Ausschlusskriterien überprüft. Das neue System erfordert eine elektronische Führung aller Praxisunterlagen und bedeutet einen beträchtlichen administrativen Mehraufwand. Beabsichtigt ist eine jederzeit mögliche standardisierte Auswertung aller codierten Daten, was bereits mit allen Softwareherstellern des Landes abgesprochen wurde.

Organisatorische Indikatoren bewerten fünf Kategorien mit maximal 184 Punkten. Führung der Krankengeschichte (85 Punkte), Kommunikation mit Patienten (8 Punkte), Auswertungen der Praxisarbeit (29 Punkte), Medikamentenabgabe (42 Punkte), Praxismanagement (20 Punkte). Patientenbewertungen ergeben bis zu 70 Punkte, die Konsultationsdauer bis 30 Punkte. Letzteres ist ein Ansporn für Praktiker mit Routinekonsultationen im 10-Minuten-Abstand. Dann gibt es Zusatzpunkte für den Abstrich nach Papanicolaou (36), Kinderimpfungen, Mütter- und Antikonzeptionsberatungen. 50 Extrapunkte für gute Erreichbarkeit, 130 Extrapunkte für im Quervergleich besonders herausragende Praxen.

\section{Grenzen und Konsequenzen}

Ein Punkt ist mit rund Fr. 108.- bewertet. Da eine Durchschnittpraxis 5500 eingeschriebene Patienten und drei Ärzte zählt, ist gemäss Autor und aktuellem Wechselkurs ab 2005 ein Zusatzverdienst für jeden Praktiker von rund Fr. 40 000.möglich. 
Ein zentraler Datenpool wird es der Regierung erlauben, die Rahmenbedingungen und Indikatorwerte dauernd anzupassen. Für die Auswertung der Daten stehen universitäre Institutionen zur Verfügung. Absehbare Folgen dieses aufwendigen Experimentes werden schon heute diskutiert: zunehmende Abhängigkeit von Computersystemen; steigende Bedeutung von Praxisschwestern; mehr Spezialkliniken für chronische Krankheiten; spezialisierte Grundversorger; vermehrte biomedizinische Ausrichtung; fraglich bessere Gesundheitsresultate; eine Fragmentierung der Grundversorgung und damit Probleme für Mehrfachkranke; weniger umfassende Betreuung; eine Vernachlässigung von allem, was nicht speziell belohnt wird; eine Zunahme der administrativen Kosten und, nie ganz auszuschliessen, Betrug.

Gute Scores bedeuten noch lange nicht gute Qualität. Da nur wenige Bereiche messbar sind, könnte sogar eine Qualitätsabnahme resultieren. Dem Schlusssatz des Autors wird man, im Jahr eins des TARMED, zustimmen: «Many countries will look with interest at the research being conducted on the new contract in the United Kingdom to see which of its anticipated benefits and harmful effects will be realized.»

- Roland M. Linking physicians' pay to the quality of care - a major experiment in the United Kingdom. N Engl J Med 2004; 351(14):1448-54.

\title{
Früherfassung von Arbeitsunfähigkeit: Was bringt die 5. IV-Revision?
}

\author{
Gedanken zum Artikel von C. L. Bohny [1]
}

\author{
C. Benz-Scheurer
}

\begin{abstract}
Als Sozialpädagoge, Schreiner und Mann einer Ärztin setze ich mich täglich praktisch mit dem Thema IV auseinander. In Basel leite ich eine Gruppe von 10 Personen in einer geschützten Werkstatt mit psychisch Kranken, die erst kürzlich auf Anraten des Kantons hin erweitert wurde. Nun steht uns durch des Bundes Sparmassnahmen das Wasser am Hals. Wenn die IV mündlich Versprochenes wahr macht, können wir bei nüchterner Betrachtung schlicht und einfach schliessen. Die getätigten Subventionen würden zu Staub zerfallen.
\end{abstract}

1 Bohny CL. Früherfassung von Arbeitsunfähigkeit: Was bringt die 5. IV-Revision? Schweiz Ärztezeitung 2004;85(38):2031-3.

Korrespondenz:

Christoph Benz-Scheurer

Sierenzerstrasse 70

CH-4055 Basel

E-Mail: chrisilbenz@freesurf.ch
Damit stünden etwa 40 IV-Bezüger mit Arbeit auf der Strasse oder kehren in die Psychiatrische Klinik zurück, und einige Sozialausgebildete landen auf dem Arbeitsamt. Sensationelle Sparrechnung! Ein Departement saniert sich auf Kosten eines andern!

Der Artikel zu den Vorbereitungen der 5. IVRevision ist für mich Anlass, einmal Gedanken von einer Front zu Gehör kommen zu lassen, die sonst ungehört bleiben: Für mich sind die vielen IV-Rentner vor allem ein Symptom einer kranken Gesellschaft ohne Krankheitseinsicht.

Im Kanton BS wird dieses Bild noch zusätzlich verzerrt, weil BS und BL zwar eine Region, aber zwei Kantone sind. Nun wird die IV-Stelle
BS ins Gebet genommen, weil sie zu viele Renten spräche, dabei bietet sie pro Kopf im Vergleich zu BL mehr geschützte Plätze an, und zudem kennen gewisse Einrichtungen in BS auch einen Wohnsitzzwang, wenn man zu ihnen kommen will. Man muss zum Teil also zügeln, um in Genuss einer Zentrumsleistung zu kommen, und entsprechend sieht die Bilanz aus.

Die auf Gewinnmaximierung orientierte Wirtschaft lagert ihre schwächsten Arbeitnehmer ins sozial nächsttiefere Segment aus, die Dividenden an die Aktionäre werden (sogar kapitalgewinnsteuerfrei) immer höher, und die Ausgaben des Sozialwesens wachsen ins Unendliche. Wenn nun die AHV/IV-Lohnprozente angehoben werden, klagt dieselbe Wirtschaft über belastende Abgaben und Standortnachteile. Sie verlagert ihre Produktivität ins Ausland, Gewinn und Dividenden werden erneut grösser, Stellen gehen verloren, und die Spirale dreht sich weiter.

In diesem Bild hat man nicht den Eindruck, dass es alles nur so kommen konnte, weil der Sozialstaat aufgeblasen sei oder weil wir zu viele faule Invaliden hätten. (Ich verzichte hier bewusst auf den Begriff «Scheininvaliden».) Wieso 
dreht man den Geldhahn eigentlich beim BSV und der IV zu und lässt gleichzeitig Quellen wie Kapitalgewinnsteuer, steilere Steuerprogression für Superverdiener und ähnliches unangetastet und verschlossen?

Diese Praxis erweckt den Eindruck, als sei der Sozialstaat schuld an einem gesellschaftlichen Problem, das niemand zu lösen imstande zu sein scheint! Auf jeden Fall treffen die neusten Sparmassnahmen nicht die armen und reichen Vielfrasse der Gesellschaft, sondern die gut funktionierenden Institutionen und die IV-Bezüger mit Pep:

- Wer nicht arbeitet, weil er wirklich nicht dazu imstande ist oder weil er nicht arbeiten will, den trifft es nicht. Er bekommt seine Rente ja auch ohne Anstrengung.

- Wer seine Arbeitsprozente bewusst so tief hält, dass er noch unter dem Lohnfreibetrag der IV liegt und noch manches via Ergänzungsleistungen (Fitnessstudio) bezahlt bekommt, wird für seine Faulheit auch nicht bestraft. (Notabene: Das ist keine Polemik. Das sind Aussagen von mir gutbekannten ehrlichen IV-Bezügern.)

- Wer arbeitet, weil er wieder erstarken will, dem wird eventuell nun auch sein geschützter Arbeitsplatz vom Staat wegrationalisiert. Wenn nicht, kann er arbeiten, wird qualitativ und quantitativ besser und bekommt einen höheren Leistungslohn (!). Damit kommt er bald in den Bereich, wo die IV viel höhere Abzüge macht, die EL gewisse Wünsche nicht mehr berücksichtigt und er selbst wieder innerhalb des Systems Schwächere mitträgt. Unter dem Strich verdient er oder sie aber oft weniger als früher, und die emotionale Kurzsichtigkeit oder bloss Faulheit führen zur Schlussfolgerung: «Es lohnt sich nicht, wenn ich mich anstrenge!» Auf diese Weise sind uns letztes Jahr mehrere Mitarbeiter abgewandert, und andere strengen sich deutlich weniger an! Das darf doch nicht wahr sein! Wir Sozialarbeitenden stossen uns sehr an diesem Missstand, weil unsere Anstrengungen in Richtung Reintegration damit durch ein Lohnsystem zur Farce werden. Es muss sich für IV-Bezüger lohnen, (mehr) zu arbeiten!

Die Ziele in der IV-Lohnberechnung sind klar und richtig, aber die Rechnung ist pädagogisch falsch - und züchtet so tatsächlich Schlendrian. Erziehung funktioniert bei vielen IV-Bezügern ebenfalls übers Portemonnaie. Auch diese Mäuse fängt man mit Speck. Dafür bleiben am Schluss dem Staat mehr «Mäuse».

Es soll hier nicht der Eindruck entstehen, alle IV-Bezüger würden mit Lohnköder gesund. Vielen ist damit nicht geholfen. Aber die faulen werden garantiert wacher, wenn sie mehr Geld verdienen können. Damit rutschen sie eventuell später in einen tieferen Unterstützungsgrad, den sie aber wiederum durch höheren Lohn ausgleichen können müssten.

Das IV-Lohnraster ist im Prinzip nicht schlecht, aber solche Fehlmechanismen müssen unbedingt korrigiert werden. Das ist meine dringende Bitte. Die Abrechnung muss natürlich so umgestaltet werden, dass fähige Mitarbeiter gefördert werden, ohne dass wirklich Schwache in finanzielle Not geraten. Bei den Abklärungen sind wir «Sozialen» gerne zur interdisziplinären Zusammenarbeit mit den Ärzten und der IVStelle bereit!

Andererseits müssen wir als Gesellschaft die ganze Situation auch als Ergebnis der uns innewohnenden Habsucht verstehen. Die Veränderung dieser Herzenshaltung dürfte tiefgreifender und schwieriger sein als eine 5. IV-Revision. Selbsterkenntnis ist der beste Weg zur Besserung. Reiche Menschen sind an sich kein Problem, nur sollten sie ihr Geld ausgeben. Es ist zum Teilen da. Es wäre allen geholfen - auch ihnen. 